\title{
EQUIVARIANT NIELSEN THEORY
}

\author{
PETER WONG \\ Department of Mathematics, Bates College \\ Lewiston, ME 04240, U.S.A. \\ E-mail: pwong@abacus.bates.edu
}

The celebrated Lefschetz fixed point theorem gives a sufficient condition, namely that the Lefschetz number $L(f)$ is nonzero, for the existence of a fixed point of a selfmap $f: X \rightarrow X$ on a compact connected polyhedron $X$. It is well known that if $X$ is a simply connected manifold then $L(f) \neq 0$ is also necessary. In other words, the converse of the Lefschetz theorem holds in this case or equivalently, $L(f)=0$ implies that $f$ is deformable to be fixed point free. For the non-simply connected case, one needs to replace $L(f)=0$ with $N(f)=0$ where $N(f)$ denotes the Nielsen number of $f$. It follows from a classical result of Wecken that $N(f)=0$ is sufficient to deform $f$ to a fixed point free map when $X$ is a manifold of dimension $\operatorname{dim} X \geq 3$.

In the category of $G$-spaces and $G$-maps where $G$ is a compact Lie group, the problem of equivariantly deforming a $G$-map to be fixed point free is more complicated. Since every $G$-space is made up of subspaces of various isotropy types $(G / H)$ (or simply $(H)$ ), the fixed points of a $G$-map $f$ is a disjoint union of orbits of fixed points of different types. For each closed subgroup $H \leq G$, the Weyl group $W H=N H / H$ acts on $X^{H}=\{x \in$ $X \mid \sigma x=x, \forall \sigma \in H\}$ and $f^{H}:=f \mid X^{H}$ is a selfmap of $X^{H}$. Moreover, if $h$ is $G$-homotopic to $f$ then $h^{H}$ and $f^{H}$ are homotopic in $X^{H}$. Therefore, the vanishing of all $N\left(f^{H}\right)$ is necessary for deforming $f$ equivariantly to be fixed point free. Fadell and Wong $[\mathrm{FW}]$ showed that $\left\{N\left(f^{H}\right)=0\right\}$ is also sufficient under some codimension hypotheses. This result was also proven independently by Borsari and Gonçalves [BoG] using Bredon's equivariant obstruction. We should point out that this result in the simply connected case ( $X^{H}$ is simply connected) was proven by Wilczyński [Wi] and independently by Vidal $[\mathrm{V}]$ using equivariant obstruction. The main idea in $[\mathrm{FW}]$ is to organize the fixed points

1991 Mathematics Subject Classification: Primary 55M20; Secondary 57S99.

Key words and phrases: Nielsen number, degree, root theory, homogeneous space, fixed point theory, equivariant maps.

Partially supported by Bates College and the Universidade de São Paulo.

The paper is in final form and no version of it will be published elsewhere. 
of a $G$-map $f$ into orbits and to partition Fixf $=\{x \in X \mid f(x)=x\}$ into equivariant fixed point classes each of which is a disjoint union of orbits. A stepwise induction on the isotropy types $(H)$ and the vanishing of $N\left(f^{H}\right)$ allow us to deform $f$ equivariantly to be fixed point free.

Nielsen fixed point theory for equivariant maps was studied in [W4] and further developed in [W5] in which techniques from relative Nielsen fixed point theory were employed. Moreover, its relationship with the Nielsen theory for periodic points was established in [W5]. (See also the survey article [W1].) On the other hand, Nielsen fixed point theory has been generalized to coincidence theory by Schirmer [S] and to root theory by Brooks [B]. Recently, equivariant Nielsen fixed point theory of [W4] has been extended to coincidences of $G$-maps by Fagundes in [Fa].

One of the central problems in Nielsen fixed point theory is to find useful computational means for calculating the Nielsen number $N(f)$. Under the so-called Jiang condition (see $[\mathrm{J}]$ ) on a space $X$, every selfmap $f: X \rightarrow X$ satisfies one of the following

$$
\begin{aligned}
& L(f)=0 \Rightarrow N(f)=0 ; \\
& L(f) \neq 0 \Rightarrow N(f)=R(f)
\end{aligned}
$$

where $R(f)$ denotes the Reidemeister number of $f$ which is defined algebraically at the fundamental group level. Thus, $(C 2)$ reduces the calculation of $N(f)$ to that of $R(f)$. (See also section II of $[\mathrm{Br}]$.)

The purpose of this paper is to give a brief summary of some results in [W2] and [W3]. We illustrate how equivariant Nielsen theory can be used to obtain results in nonequivariant Nielsen fixed point theory. More precisely, we use an equivariant analog of the Nielsen root theory to show the following which is a special case of a more general result in [W2].

TheOREM A. Let $G$ be a compact connected Lie group, $K$ a finite subgroup and $M=$ $G / K$ the homogeneous space of left cosets. For any $f: M \rightarrow M$, either

(1) $L(f)=0 \Rightarrow N(f)=0$; or

(2) $L(f) \neq 0 \Rightarrow N(f)=R(f)$.

Furthermore, $L(f)=0$ implies that $f$ is homotopic to a fixed point free map.

I would like to thank Daciberg Gonçalves for many stimulating conversations and for his kind invitation, during the summer of 1996, to the Instituto de Matemática e Estatística of the Universidade de São Paulo at which the present work was completed. I would also like to thank the Banach Center for its hospitality.

1. Equivariant Nielsen root theory. We first review the Nielsen root theory introduced by Brooks in [B] (see also [K]). Given a map $\varphi: X \rightarrow Y$ between two compact connected topological spaces and a point $a \in Y$. The solutions of the equation $\varphi(x)=a$ are called the roots of $\varphi$ and the set of roots is denoted by $\Gamma(\varphi)$. Given $x_{1}, x_{2} \in \Gamma(\varphi)$, we say that $x_{1}$ and $x_{2}$ are Nielsen equivalent as roots with respect to $\varphi$ if there exists a path $C:[0,1] \rightarrow X$ with $C(0)=x_{1}, C(1)=x_{2}$ such that $\varphi \circ C$ is homotopic to the constant map $\bar{a}$ at $a$ rel the endpoints. Let $\widetilde{\Gamma_{\varphi}}$ be the set of equivalence (root) classes. 
Given $\alpha \in \widetilde{\Gamma_{\varphi}}, \alpha$ is said to be inessential if there exists a neighborhood $U$ and a homotopy $f \sim_{H_{t}} \varphi$ relative to $X \times[0,1]-U$ such that $U \cap \Gamma(\varphi)=\alpha, H^{-1}(a) \cap U$ is compact in $U$ and $\Gamma\left(H_{1}\right) \cap U=\emptyset$. Otherwise, $\alpha$ is essential. We define the Nielsen (root) number of $\varphi$ to be

$$
N(\varphi ; a):=\#\{\text { essential root classes of } \varphi\} .
$$

In $[\mathrm{B}]$, Brooks showed the following

THEOREM 1. If $Y$ is a manifold then either $N(\varphi ; a)=0$ or $N(\varphi ; a)=R(\varphi ; a):=$ $\#\left[\pi_{1}(Y) / \operatorname{Im}\left(\varphi_{\sharp}\right)\right]$ where $\varphi_{\sharp}: \pi_{1}(X) \rightarrow \pi_{1}(Y)$ is the induced homomorphism on fundamental groups.

If $X$ and $Y$ are closed orientable $n$-manifolds, then a numerical (root) index $\omega(\varphi ; \alpha)$ can be defined. Moreover, we have $([\mathrm{B}])$

THEOREM 2. (1) All root classes of $\varphi$ have the same index.

(2) $\sum_{\alpha \in \widetilde{\Gamma_{\varphi}}} \omega(\varphi ; a)=\operatorname{deg} \varphi$.

(3) $N(\varphi ; a)>0 \Rightarrow N(\varphi ; a)=R(\varphi ; a)$.

(4) If $n \geq 3$, then $N(\varphi ; a)=0 \Rightarrow \varphi \sim f$ such that $\Gamma(f)=\emptyset$.

Suppose that $G$ is a compact Lie group, $X$ and $Y$ are both compact $G$-ENRs. Let $\mathcal{F}=\{(H) \in I s o(X) \cup I s o(Y)|| W H \mid<\infty\}$ where $I s o(Z)$ denotes the set of isotropy types of a $G$-space $Z$. We assume that $Y^{G} \neq \emptyset, X^{H}$ and $Y^{H}$ are connected (if not empty) for each $(H) \in \mathcal{F}$. Choose a point $a \in Y^{G}$. Let $\varphi: X \rightarrow Y$ be a $G$-map. For any $(H) \in \mathcal{F}, \varphi^{H}$ : $X^{H} \rightarrow Y^{H}$ is a $W H$-map. Given $x_{1}, x_{2} \in \Gamma_{W H}\left(\varphi^{H}\right):=\left\{x \in X^{H} \mid \varphi^{H}(x)=a\right\}$, we say that $x_{1}$ and $x_{2}$ are $W H$-Nielsen equivalent if either $x_{2}=\gamma x_{1}$ for some $\gamma \in W H$ or there exists a path $C:[0,1] \rightarrow X^{H}$ such that $C(0)=x_{1}, C(1)=\sigma x_{2}$ for some $\sigma \in W H$ and $\varphi^{H} \circ C \sim \bar{a}\left(\right.$ in $\left.Y^{H}\right)$ rel the endpoints. Denote by $\widetilde{\Gamma_{\varphi^{H}}}$ the set of $W H$-(root) equivalence classes. Given $\alpha \in \widetilde{\Gamma_{\varphi^{H}}}$, we say that $\alpha$ is inessential if there exists a $W H$-invariant neighborhood $U$ in $X^{H} \times[0,1]$ and a $W H$-homotopy $F: X^{H} \times[0,1] \rightarrow Y^{H}$ such that $F_{0}=\varphi^{H}, U \cap \Gamma\left(\varphi^{H}\right)=\alpha, F^{-1}(a) \cap U$ is compact in $U$ and $U \cap \Gamma\left(F_{1}\right)=\emptyset$. Otherwise, $\alpha$ is said to be essential. Define the $W H$-Nielsen root number to be

$$
N_{W H}\left(\varphi^{H} ; a\right):=\#\left\{\text { essential } W H \text {-essential root classes of } \varphi^{H}\right\} \text {. }
$$

Similarly, one can define a $W H$-Reidemeister number as follows.

For any $(H) \in \mathcal{F}$, let $\eta_{X^{H}}: \tilde{X^{H}} \rightarrow X^{H}$ and $\eta_{Y^{H}}: \tilde{Y^{H}} \rightarrow Y^{H}$ be the universal coverings of $X^{H}$ and $Y^{H}$, respectively. Let

$$
\begin{aligned}
& \hat{G}\left(X^{H}\right):=\left\{\sigma \in \operatorname{Homeo}\left(\tilde{X^{H}}\right) \mid \eta_{X^{H}} \circ \sigma=\bar{\sigma} \circ \eta_{X^{H}}, \bar{\sigma} \in W H\right\} ; \\
& \hat{G}\left(Y^{H}\right):=\left\{\sigma \in \operatorname{Homeo}\left(\tilde{Y^{H}}\right) \mid \eta_{Y^{H}} \circ \sigma=\bar{\sigma} \circ \eta_{Y^{H}}, \bar{\sigma} \in W H\right\} .
\end{aligned}
$$

It follows that we have the following exact sequences of groups:

$$
\begin{gathered}
1 \rightarrow \pi_{1}\left(X^{H}\right) \rightarrow \hat{G}\left(X^{H}\right) \rightarrow W H \rightarrow 1, \\
1 \rightarrow \pi_{1}\left(Y^{H}\right) \rightarrow \hat{G}\left(Y^{H}\right) \rightarrow W H \rightarrow 1 .
\end{gathered}
$$

The map $\varphi^{H}: X^{H} \rightarrow Y^{H}$ induces a homomorphism on fundamental groups and hence a homomorphism $\varphi_{H}: \hat{G}\left(X^{H}\right) \rightarrow \hat{G}\left(Y^{H}\right)$. Let $\tilde{a} \in \eta_{Y^{H}}^{-1}(a)$. Since $\bar{\sigma} a=a$ for all $\bar{\sigma} \in W H$, 
there is a unique homomorphism $\tau_{H}: W H \rightarrow \hat{G}\left(Y^{H}\right)$ such that $\tau_{H}(\bar{\sigma})(\tilde{a})=(\tilde{a})$ and $\tau_{H}(\bar{\sigma})$ covers $\bar{\sigma}$. In particular, if $\bar{\sigma}$ is the identity in $W H, \tau_{H}(\bar{\sigma})=1_{Y^{H}}$. Then, $\hat{G}\left(X^{H}\right)$ acts on $\pi_{1}\left(Y^{H}\right)$ via

$$
\sigma \cdot \beta=\tau_{H}(\bar{\sigma}) \beta \varphi_{H}(\sigma)^{-1}
$$

where $\sigma \in \hat{G}\left(X^{H}\right), \sigma$ covers $\bar{\sigma}$ and $\beta \in \pi_{1}\left(Y^{H}\right)$.

We define the $W H$-Reidemeister (root) number of $\varphi^{H}$ to be the number of orbits of this action and denote it by $R_{W H}\left(\varphi^{H} ; a\right)$.

Under appropriate hypotheses ([W3]), equivariant analogs of Theorem 1 and of Theorem 2 are proven. Furthermore, $\varphi \sim_{G} f$ with $\Gamma(f)=\emptyset$ if and only if $\operatorname{deg} \varphi^{H}=0$ for each $(H) \in \mathcal{F}$. For the purpose of our application in section 2, we need only the following special case.

Theorem 3. Let $X$ and $Y$ be closed orientable smooth $K$-manifolds of dimension $n$ where $K$ is a finite group. Suppose that $K$ acts on $X$ and on $Y$ as orientation preserving homeomorphisms and $K$ acts freely on $X$. Let $a \in Y^{K} \neq \emptyset$ and $\varphi: X \rightarrow Y$ be a $K$-map. Then

(1) $\operatorname{deg} \varphi \equiv 0 \bmod |K|$;

(2) $\operatorname{deg} \varphi=0 \Rightarrow N_{K}(\varphi ; a)=0$;

(3) if $\operatorname{deg} \varphi \neq 0$ then all $K$-root classes of $\varphi$ are essential; their root indices have the same sign and $N_{K}(\varphi ; a)=R_{K}(\varphi ; a)$.

The basic idea in proving Theorem 3 is to use Theorem 2 together with the concept of orbits as in equivariant Nielsen fixed point theory. Without loss of generality, we may assume that $\# \Gamma(\varphi)<\infty$. Then $\Gamma(\varphi)$ is partitioned into $K$-root classes $R_{1}, \ldots, R_{m}$. It follows from the definition of $R_{i}$ that if $\alpha$ is an ordinary root class of $\varphi$ (forget the $K$-equivariance) then there exists a unique $j, 1 \leq j \leq m$ such that $\alpha \subset R_{j}$. In other words, each $R_{j}$ is a disjoint union of ordinary root classes. On the other hand, for each $j$, $R_{j}$ is a disjoint union $\sqcup \mathcal{O}_{j_{r}}$ of $K$-orbits of roots. Since $K$ acts freely on $X$ as orientation preserving homeomorphisms, the root index of each point on a single $K$-orbit $\mathcal{O}_{j_{r}}$ is the same. Thus, by (2) of Theorem 2, $|K|$ must divide $\operatorname{deg} \varphi$ and hence (1) is established. It follows from (1) of Theorem 2 that $\operatorname{deg} \varphi=0$ implies that every ordinary root class of $\varphi$ has zero root index. Thus, each $R_{j}$ has zero root index and hence is inessential. This proves $(2)$. In the case when $\operatorname{deg} \varphi \neq 0$, every $R_{j}$ has root index equal to a positive integral multiple $k_{j}$ of $\omega$ where $\omega$ is the root index of a single ordinary root class of $\varphi$. Since $k_{j}$ may vary with $j, 1 \leq j \leq m$, we conclude only that the root index of $R_{j}$ have the same sign. The assertion $N_{K}(\varphi ; a)=R_{K}(\varphi ; a)$ is similar to (3) of Theorem 2 .

2. Application to homogeneous spaces. Let $G$ be a compact connected Lie group and $K$ a finite subgroup. The homogeneous space $M=G / K$ of left cosets is an orientable manifold. The subgroup $K$ acts freely on $G$ via $k \circ g=g k^{-1}$ and on $M$ via $k * g K=k g K$. Fadell observed $[\mathrm{F}]$ that for every map $f: M \rightarrow M$, there is an associated $K$-map $\varphi: G \rightarrow M$ given by $\varphi(g)=g^{-1} f(g K)$. Conversely, given a $K$-map $\varphi: G \rightarrow M$, we associate to it a map $f: M \rightarrow M$ given by $f(g K)=g \varphi(g)$. Thus, $f(g K)=g K$ if and only if $\varphi(g)=e K$ where $e \in G$ is the identity element in $G$. In fact, we have 
THEOREM 4 ([W2]). There is a 1-1 correspondence between the fixed point classes of $f$ and the $K$-root classes of $\varphi$. Furthermore, $R(f)=R_{K}(\varphi ; e K)$ where $R(f)$ denotes the Reidemeister number of $f$.

We now give a sketch of proof of Theorem A.

CAse I. Suppose $\operatorname{dim} M \geq 3$. Without loss of generality, we may assume that \#Fixf = $N(f)$. Let Fixf $=\left\{g_{1} K, \ldots, g_{m} K\right\}$ where $g_{i} \in G$ and $m=N(f)$. By Theorem 4, the $K$-map $\varphi$, which corresponds to $f$, has $\mathcal{O}_{1}, \ldots, \mathcal{O}_{m}$ as $K$-root classes where $\mathcal{O}_{i}$ is the $K$-orbit of $g_{i}, i=1, \ldots, m$. A straightforward calculation shows that the fixed point index $i\left(f, g_{j} K\right)$ coincides with the numerical root index $\omega\left(\varphi ; g_{j}\right)$. Since the $K$-action on $G$ is orientation preserving, it follows that $\omega\left(\varphi ; g_{j}\right)=\omega\left(\varphi ; k \circ g_{j}\right)$ for all $k \in K$. By (2) of Theorem 2, we conclude that

$$
\operatorname{deg} \varphi=\sum_{j=1}^{m}|K| \cdot \omega\left(\varphi ; g_{j}\right)
$$

By (3) of Theorem $3, \operatorname{deg} \varphi \neq 0$ if and only if all $\omega\left(\varphi ; g_{j}\right)$ are nonzero and have the same sign. That is, $\operatorname{deg} \varphi \neq 0$ if and only if all $i\left(f, g_{j} K\right)$ are nonzero and have the same sign. This is equivalent to $L(f) \neq 0$. Therefore, $\mathcal{O}_{j}$ is essential as a $K$-root class of $\varphi$ if and only if $g_{j} K$ is essential as a fixed point class of $f$. In other words, $N_{K}(\varphi ; a)=N(f)=m$. It follows from (3) of Theorem 3 that $L(f)=0 \Rightarrow N(f)=0$ and $L(f) \neq 0 \Rightarrow N(f)=R(f)$. In the case when $L(f)=0, N(f)=0$ implies that $f$ is deformable to be fixed point free.

CAse II. Suppose $\operatorname{dim} M=2$. Then $M$ is the torus and the results are well-known.

Corollary B. For any selfmap $f: M \rightarrow M$,

$$
|K| \cdot L(f)=\operatorname{deg} \varphi
$$

where $\varphi: G \rightarrow M$ is the corresponding $K$-map.

Proof. This follows directly from $(*)$ since

$$
\sum_{j=1}^{m} \omega\left(\varphi, g_{j}\right)=\sum_{j=1}^{m} i\left(f, g_{j} K\right)=L(f) \text {. }
$$

Corollary B generalizes a result of Duan [Du] in which the formula $L(f)=\operatorname{deg} \varphi_{f}$ was proven, where $f: G \rightarrow G, \varphi_{f}(g)=g^{-1} f(g)$ and $G$ is a compact connected Lie group.

REMARK 1. In [W2], we show that Theorem A holds in general for any closed subgroup $K$ with $M=G / K$ orientable and $p_{*}: H_{n}(G) \rightarrow H_{n}(M)$ nonzero where $n=\operatorname{dim} M$. The technique used in Theorem A cannot be readily extended to coincidences of two selfmaps on $M$. A different approach using $\mathcal{C}$-nilpotent actions has been devised in $[\mathrm{GW}]$ so that Theorem $\mathrm{A}$ is extended to coincidences of a pair of maps $f_{1}, f_{2}: M \rightarrow M$ and hence an alternative proof of Theorem $\mathrm{A}$ is given.

\section{References}

[BoG] L. Borsari and D. GonÇALVEs, G-deformation to fixed point free maps via obstruction theory, unpublished (1989). 
[B] R. BRooks, Certain subgroups of the fundamental group and the number of roots of $f(x)=a$, Amer. J. Math. 95 (1973), 720-728.

[Br] R. F. Brown, Nielsen fixed point theory on manifolds, these proceedings.

[Du] H. DuAn, The Lefschetz number of selfmaps of Lie groups, Proc. Amer. Math. Soc. 104 (1988), 1284-1286.

[F] E. FADELL, Two vignettes in fixed point theory, in: Topological Fixed Point Theory and Applications (Tianjin, 1988), B. Jiang (ed.), Lecture Notes in Math. 1411, Springer, Berlin, 1989, 46-51.

[FW] E. FAdell and P. Wong, On deforming G-maps to be fixed point free, Pacific J. Math. 132 (1988), 277-281.

[Fa] P. FAGundes, Equivariant Nielsen coincidence theory, in: 10th Brazilian Topology Meeting (São Carlos, 1996), P. Schweitzer (ed.), Matemática Contempor̂anea 13, Sociedade Brasileira de Matemática, Rio de Janeiro, 1997, 117-142.

[GW] D. Gonçalves and P. Wong, Homogeneous spaces in coincidence theory, in: 10th Brazilian Topology Meeting (São Carlos, 1996), P. Schweitzer (ed.), Matemática Contempor̂anea 13, Sociedade Brasileira de Matemática, Rio de Janeiro, 1997, 143-158.

[J] B. JiAng, Lectures on Nielsen Fixed Point Theory, Contemp. Math. 14, Amer. Math. Soc., Providence, 1983.

[K] T. KIang, The Theory of Fixed Point Classes, Science Press, Springer, Berlin-Beijing, 1989.

[S] H. SchiRmer, Mindestzahlen von Koinzidenzpunkten, J. Reine Angew. Math. 194 (1955), 21-39.

[V] A. VIDAL, Äquivariante Hindernistheorie für G-Deformationen, Dissertation, Universität Heidelberg, Heidelberg, 1985.

[Wi] D. Wilczyński, Fixed point free equivariant homotopy classes, Fund. Math. 123 (1984), 47-60.

[W1] P. Wong, Equivariant Nielsen fixed point theory and periodic points, in: Nielsen Theory and Dynamical Systems (Mt. Holyoke, 1992), C. McCord (ed.), Contemp. Math. 152, Amer. Math. Soc., Providence, 1993, 341-350.

[W2] P. Wong, Fixed point theory for homogeneous spaces, Amer. J. Math. 120 (1998), 23-42.

[W3] P. Wong, Root theory for G-maps, in preparation.

[W4] P. Wong, Equivariant Nielsen fixed point theory for G-maps, Pacific J. Math. 150 (1991), 179-200.

[W5] P. Wong, Equivariant Nielsen numbers, Pacific J. Math. 159 (1993), 153-175. 